\title{
Control of $\mathrm{N}_{2}^{+}$air lasing
}

\author{
Mathew Britton $\odot,{ }^{1, *}$ Marianna Lytova, ${ }^{1}$ Dong Hyuk Ko $\odot,{ }^{1}$ Abdulaziz Alqasem,${ }^{1,2}$ Peng Peng $\odot,{ }^{1}$ D. M. Villeneuve,, 3 \\ Chunmei Zhang, ${ }^{1, \dagger}$ Ladan Arissian, ${ }^{1,3,4}$ and P. B. Corkum ${ }^{1, \ddagger}$ \\ ${ }^{1}$ Department of Physics, University of Ottawa, Ottawa, Canada K1N 6N5 \\ ${ }^{2}$ Department of Physics, King Saud University, Riyadh 11451, Saudi Arabia \\ ${ }^{3}$ National Research Council of Canada, Ottawa, Canada K1A OR6 \\ ${ }^{4}$ Center for High Technology Materials, Albuquerque, New Mexico 87106, USA
}

(Received 21 July 2020; revised 23 October 2020; accepted 26 October 2020; published 13 November 2020)

\begin{abstract}
A near-infrared laser generates gain on transitions between the $B^{2} \Sigma_{\mathrm{u}}^{+}$and $X^{2} \Sigma_{\mathrm{g}}^{+}$states of the nitrogen molecular cation in part by coupling the $X^{2} \Sigma_{\mathrm{g}}^{+}$and $A^{2} \Pi_{\mathrm{u}}$ states in the $V$ system. Traditional time resolved pump-probe measurements rely on postionization coupling by the pump pulse to initialize dynamics in the $A^{2} \Pi_{\mathrm{u}}$ state. Here we show that a weak second excitation pulse reduces ambiguity because it acts only on the ion independent of ionization. The additional control pulse can increase gain by moving population to the $A^{2} \Pi_{\mathrm{u}}$ state, which modifies the lasing emission in two distinct ways. The presence of fast decoherence on $X^{2} \Sigma_{\mathrm{g}}^{+}$to $A^{2} \Pi_{\mathrm{u}}$ transitions may prevent the formation of a coherent rotational wave packet in the ground state in our experiment, but the control pulse can reverse impulsive alignment by the pump pulse to remove rotational wave packets in the $B^{2} \Sigma_{\mathrm{u}}^{+}$state.
\end{abstract}

DOI: 10.1103/PhysRevA.102.053110

\section{INTRODUCTION}

Focusing a powerful near-infrared femtosecond laser into air reveals gain on transitions in the ultraviolet between the different vibrational states of the upper $B^{2} \Sigma_{\mathrm{u}}^{+}$and the ground $X^{2} \Sigma_{\mathrm{g}}^{+}$electronic states of the nitrogen molecular cation [1]. Pump laser pulses near $800 \mathrm{~nm}$ move population from the ground state to the middle $A^{2} \Pi_{\mathrm{u}}$ state of the ion [2-12], which initiates a vibrational wave packet that can temporarily trap population $[2,3,5]$. The $X^{2} \Sigma_{\mathrm{g}}^{+}$to $A^{2} \Pi_{\mathrm{u}}$ interaction contributes to gain by depleting the ground state, but it also enables control of the gain and emission.

Elliptical [11] and polarization-modulated [7,13,14] pump pulses exploit the postionization interaction with the $A^{2} \Pi_{\mathrm{u}}$ state to control gain, but these experiments are complicated because the pump pulse acts before, during, and after ionization. A second weak excitation pulse can reduce ambiguity because it acts only on the ion independent of ionization. Electronic and vibrational coherences produce rapid oscillations of gain with multiple probe pulses [15] or pump pulses $[9,10,16,17]$. In addition, a second $800-\mathrm{nm}$ pulse modifies gain and quenches emission, which are both consistent with the $A^{2} \Pi_{\mathrm{u}}$ state interaction [9].

We perform a pump-probe experiment on the ion itself to investigate and harness the $A^{2} \Pi_{\mathrm{u}}$ state. For clarity, we refer to the additional pulse as "control." This experiment minimizes the interaction length using a narrow gas jet in vacuum to isolate gain from the effects of propagation [18,19]. By vary-

\footnotetext{
*mathew.britton@uottawa.ca

†chunmei.zhang@uottawa.ca

‡pcorkum@uottawa.ca
}

ing both control and probe delays, we also observe modified gain and emission. While the influence of the $A^{2} \Pi_{u}$ state on gain is clear, we discuss two distinct ways that its presence modifies the emission. The control pulse also strengthens or cancels the initial rotational wave packet in the $B^{2} \Sigma_{\mathrm{u}}^{+}$state with a new conventionally generated rotational wave packet. The weakness of $X^{2} \Sigma_{\mathrm{g}}^{+}$state rotational frequencies allows us to conclude that the $A^{2} \Pi_{\mathrm{u}}$ state interaction may prevent a coherent wave packet from forming in the $X^{2} \Sigma_{\mathrm{g}}^{+}$state.

\section{RESULTS}

Figure 1 (black line) shows the gain-length product $g_{L}$ as a function of probe delay for the traditional pump-probe measurement without a control pulse. Zero delay corresponds to the arrival of the pump pulse $(\approx 35 \mathrm{fs}, 800 \mathrm{~nm})$, and the weak second harmonic probe pulse $(\approx 100 \mathrm{fs}, 400 \mathrm{~nm}$, $\left.<10^{10} \mathrm{~W} / \mathrm{cm}^{2}\right)$ measures gain at $391 \mathrm{~nm}\left[B^{2} \Sigma_{\mathrm{u}}^{+}(\nu=0) \rightarrow\right.$ $\left.X^{2} \Sigma_{\mathrm{g}}^{+}(v=0)\right]$. The rotational wave packets in the states of the ion are imprinted on the decay of gain [4,19-27].

When the weak control pulse $(\approx 100 \mathrm{fs}, 800 \mathrm{~nm})$ arrives at a fixed delay in Fig. 1, the experiment depends on the order of the three pulses: pump-probe-control and pump-controlprobe. The latter is like the traditional experiment because the probe pulse measures dynamics initiated by both the pump and control pulses. As the control intensity increases in Fig. 1, the amplitude of modulations from rotational wave packets decreases in pump-control-probe measurements. In the pump-probe-control measurements that we discuss later, the control pulse modifies the system during the emission following the probe pulse that is also known as free-induction decay. 


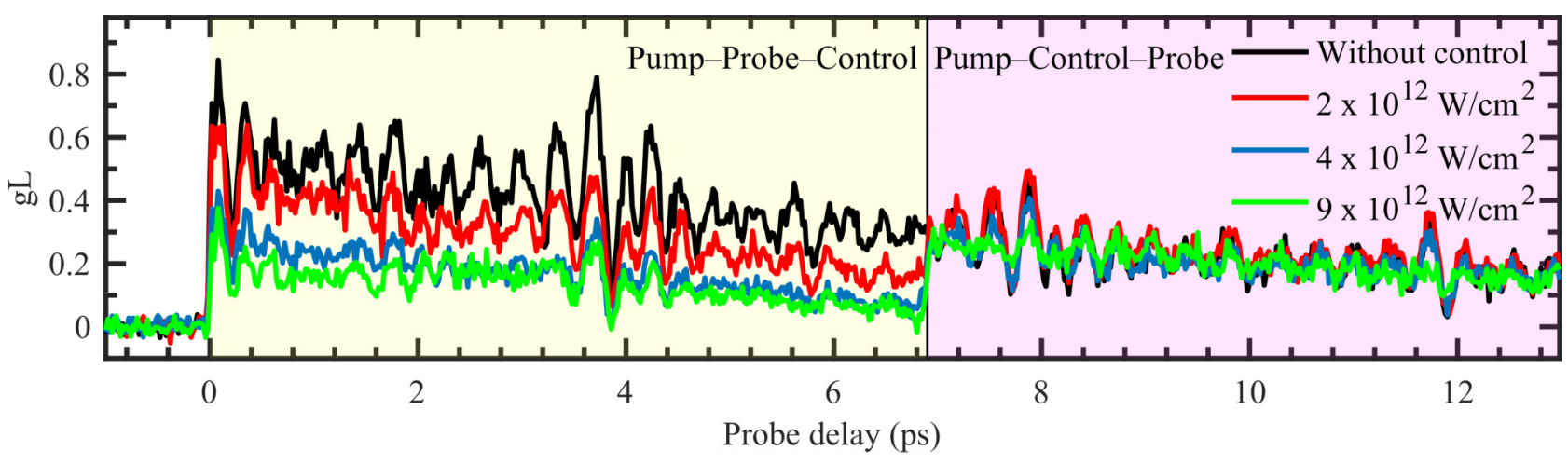

FIG. 1. The gain-length product $\left(g_{L}\right)$ as a function of pump-probe delay. The black line is without a control pulse. When the control pulse arrives near 7 ps (vertical line), the experiment depends on the ordering of the pulses. The control pulse intensity $\left(I_{\text {control }}\right)$ is in the legend. The pump intensity $\left(I_{\text {pump }}\right)$ is $1.9 \times 10^{14} \mathrm{~W} / \mathrm{cm}^{2}$.

Figure 2(a) shows the Fourier transform of the modulations without a control pulse (black line). The narrow peaks in the frequency domain identify wave packets from different electronic states. Vertical dotted lines mark the rotational frequencies for the upper $B^{2} \Sigma_{\mathrm{u}}^{+}$state, and they align well with the positions of the peaks. Conversely, few peaks align with the expected positions for the ground state of the ion and the neutral molecule, as discussed in Appendix A. The wave packet in the upper state is dominant in our experiment, which is consistent with fast decoherence in the $X^{2} \Sigma_{\mathrm{g}}^{+}$state or a large population inversion.

\section{A. Pump-control-probe}

The Fourier transform of the modulations shows how the rotational wave packets change. The red line in Fig. 2(a) is the Fourier transform of modulations when the control pulse arrives before ionization $(-0.5 \mathrm{ps})$, which is like the traditional measurement without a control pulse except for alignment of $\mathrm{N}_{2}$ before ionization [28]. As the control delay increases beyond zero in Fig. 2(b), an appropriately timed control pulse interaction periodically reduces or increases the amplitude of the $B^{2} \Sigma_{\mathrm{u}}^{+}$state rotational frequencies. To do this, the control pulse can create a new wave packet that interferes with the original [29]. This interference depends on the delay between the pump and control pulses.

If the control pulse intensity is high, Fig. 2(c) shows that the new rotational wave packet can overpower the original and add new modulations that are offset in time. At $1 \mathrm{ps}$, the amplitude of the original modulations is reduced and modulations from the control pulse wave packet emerge. This confirms the role of rotational excitation by the control pulse and demonstrates a method to modify or minimize rotational wave packets in the $\mathrm{N}_{2}^{+}$air laser. The amplitude of the exponential decay beneath the modulations is lower with the control pulse in Fig. 2(c), but we only observe this at high control intensity. At control intensities below about $3 \times 10^{13} \mathrm{~W} / \mathrm{cm}^{2}$, we usually observe higher gain (e.g., see Appendix A). This is consistent with population exchange from the $X^{2} \Sigma_{\mathrm{g}}^{+}$to $A^{2} \Pi_{u}$ states [9]. The onset of ionization and plasma defocusing may explain lower gain at high control intensity in Fig. 2(c).
Neither the pump nor control pulse generate a strong rotational wave packet in the $X^{2} \Sigma_{\mathrm{g}}^{+}$state. If the $X^{2} \Sigma_{\mathrm{g}}^{+}$state is populated, the pulses may not generate a strong or coherent wave packet because of the simultaneous interaction with the $A^{2} \Pi_{\mathrm{u}}$ state. The bandwidth of the pulses covers more than three $X^{2} \Sigma_{\mathrm{g}}^{+}$to $A^{2} \Pi_{\mathrm{u}}$ state vibronic transitions [30]. Figure 3(a) shows that the equilibrium internuclear separation of the $A^{2} \Pi_{\mathrm{u}}$ state is relatively large, so vibrational motion can temporarily trap population in the $A^{2} \Pi_{\mathrm{u}}$ state. The pulse durations exceed the timescale of vibrational dynamics and may create an unusual rotational wave packet in the $X^{2} \Sigma_{\mathrm{g}}^{+}$ state as population moves between the two states and becomes temporarily trapped. This could reduce the alignment of the $X^{2} \Sigma_{\mathrm{g}}^{+}$state. In addition, collisions between electrons and ions could incoherently mix the $X^{2} \Sigma_{\mathrm{g}}^{+}$and $A^{2} \Pi_{\mathrm{u}}$ states, which would destroy the rotational wave packet in the $X^{2} \Sigma_{\mathrm{g}}^{+}$state like the $B^{2} \Sigma_{\mathrm{u}}^{+}$state [19].

\section{B. Pump-probe-control}

These mechanisms may also contribute during pumpprobe-control measurements. Zhang et al. recently showed that a control pulse can modify the coherent emission between the $B^{2} \Sigma_{\mathrm{u}}^{+}$and $X^{2} \Sigma_{\mathrm{g}}^{+}$states by coupling the $X^{2} \Sigma_{\mathrm{g}}^{+}$and $A^{2} \Pi_{\mathrm{u}}$ states because of the shared ground state [9]. In our measurement, the probe pulse generates coherent emission at $391 \mathrm{~nm}$ $\left[B^{2} \Sigma_{\mathrm{u}}^{+}(v=0) \rightarrow X^{2} \Sigma_{\mathrm{g}}^{+}(v=0)\right]$. The control pulse reduces gain when it arrives during the emission (e.g., in Fig. 1). The transition at $785 \mathrm{~nm}\left[A^{2} \Pi_{\mathrm{u}}(v=2) \rightarrow X^{2} \Sigma_{\mathrm{g}}^{+}(v=0)\right]$ forms a $V$ system with the transition at $391 \mathrm{~nm}$, as illustrated in Fig. 3(b). Like the rotational coherence in the $X^{2} \Sigma_{\mathrm{g}}^{+}$state, an 800-nm pulse can modify the coherent emission between the $X^{2} \Sigma_{\mathrm{g}}^{+}$and $B^{2} \Sigma_{\mathrm{u}}^{+}$states by coupling the $X^{2} \Sigma_{\mathrm{g}}^{+}$and $A^{2} \Pi_{\mathrm{u}}$ states. This case is slightly different because separate pulses create and modify the coherence, and the excited states cannot directly couple.

The control pulse can modify the emission in two distinct ways that we illustrate using a numerical threelevel density-matrix calculation described in Appendix B. Figure 4(a) shows the calculated spatial profile of the probe pulse as a function of time and divergence on a logarithmic color scale without the control pulse. The superimposed lines 

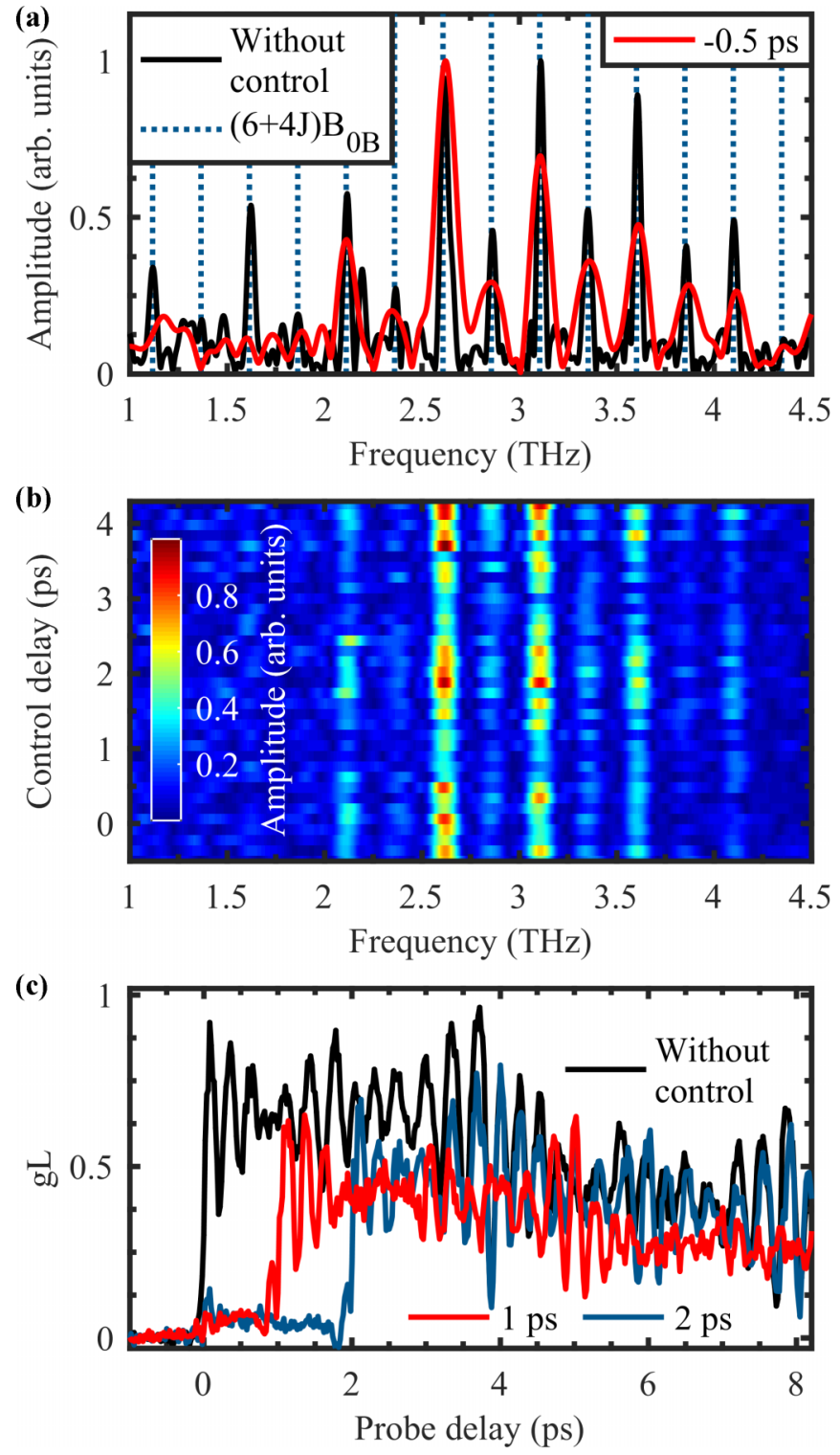

FIG. 2. Rotational wave packets. (a) The amplitude of the Fourier transform of the modulations, where frequency is the Fourier conjugate parameter of the delay between the pump and probe. The black line is without the control pulse. The vertical dashed lines indicate the rotational frequencies for the $B^{2} \Sigma_{u}^{+}$state. The lower-resolution data include the control pulse at -0.5 ps. (b) The amplitude of the modulations as a function of frequency and control delay. The peaks from the above panel oscillate as a function of control delay. $I_{\text {control }} \sim 10^{13} \mathrm{~W} / \mathrm{cm}^{2}$. (c) An intense control pulse $\left(>3 \times 10^{13} \mathrm{~W} / \mathrm{cm}^{2}\right)$ at 1 and 2 ps. $I_{\text {pump }}=2.5 \times 10^{14} \mathrm{~W} / \mathrm{cm}^{2}$.

show the magnitudes of the off-diagonal density-matrix elements. The probe pulse couples $|0\rangle$ and $|2\rangle$ weakly, which generates coherence $\rho_{02}$ and emission trailing the pulse. The phenomenological coherence time $T_{2}^{02}=5 \mathrm{ps}$ is similar to the 391-nm emission duration [4,26,31-33]

The control pulse arrives at $0.7 \mathrm{ps}$ and strongly couples $|0\rangle$ and $|1\rangle$ in Figs. 4(b) and 4(c). In Fig. 4(b), the coherence time of the transition near $800 \mathrm{~nm}$ is $T_{2}^{01}=5$ ps so the process is essentially coherent. Rabi oscillations between $|0\rangle$ and $|1\rangle$

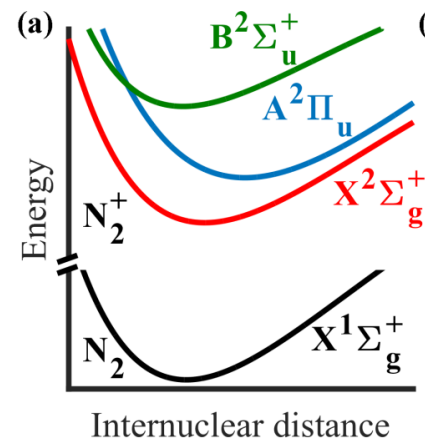

(b)

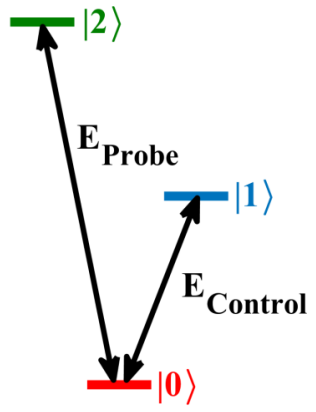

FIG. 3. Energy diagrams. (a) Potential energy of the ground state of neutral nitrogen and the $X^{2} \Sigma_{\mathrm{g}}^{+}, A^{2} \Pi_{\mathrm{u}}$, and $B^{2} \Sigma_{\mathrm{u}}^{+}$states of $\mathrm{N}_{2}^{+}$. (b) Energy levels of a $V$ system.

produce coherence $\rho_{01}$ that grows and oscillates, and they also modify $\rho_{02}$ because of the shared ground state. This induces a phase shift in $\rho_{02}$ and the resulting emission. The spatial profile of the control pulse makes the interaction nonuniform, so Rabi oscillations vary in the plane perpendicular to the propagation direction. The spatially dependent phase shift

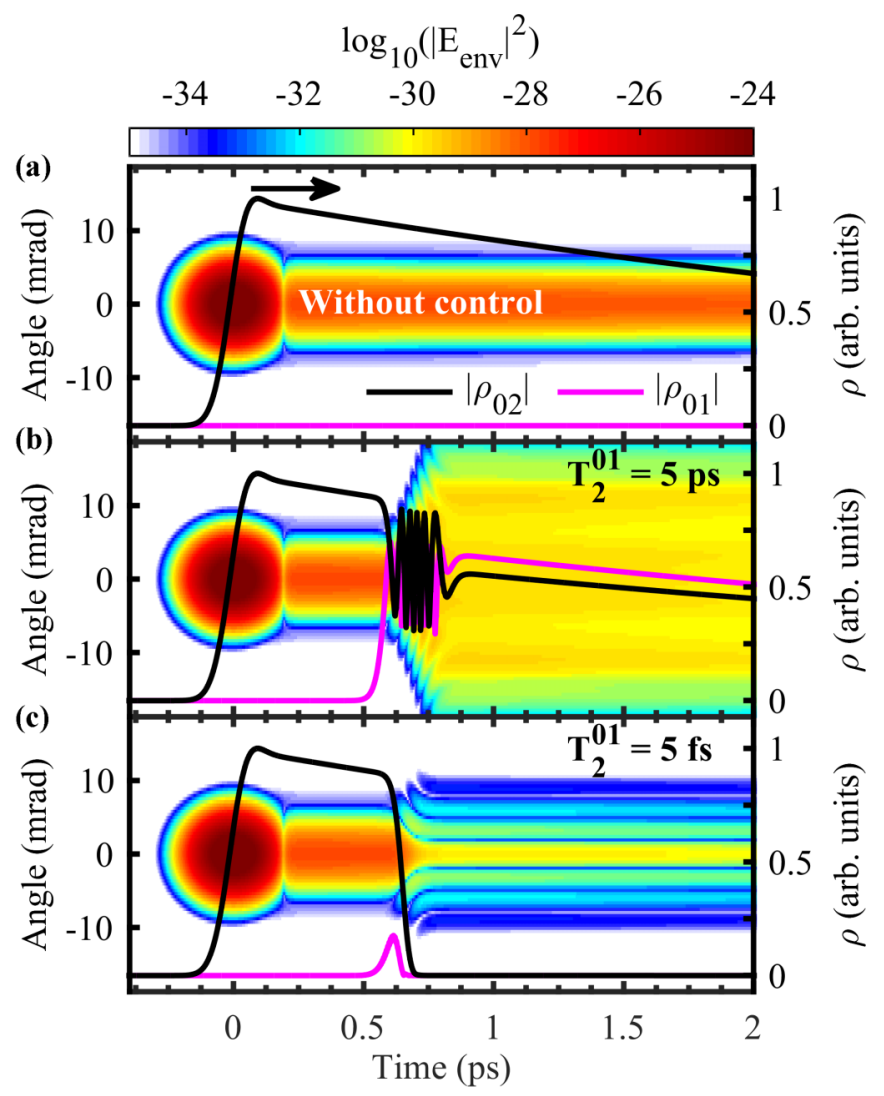

FIG. 4. Calculation results. (a) The spatial intensity profile of the emission on a logarithmic color scale without the control pulse for reference. The superimposed lines show the magnitudes of the off-diagonal density-matrix elements at the center of the beam as a function of time (right-hand axis). (b) The control pulse modifies the wavefront of the emission when it arrives at 0.7 ps. (c) Quenching dominates using $T_{2}^{01}=5 \mathrm{fs}$. 
modifies the wave front and divergence of the emission like a spatial light modulator [34]. Similarly, the Stark shift induced by a nonresonant control pulse was used to redirect extreme ultraviolet free-induction decay [35]. The phase shift due to the dynamic Stark effect was relatively small in this calculation, so that contribution is excluded.

The coherence time is $T_{2}^{01}=5 \mathrm{fs}$ in Fig. 4(c) so the control pulse generates damped Rabi oscillations between $|0\rangle$ and $|1\rangle$ and short-lived coherence $\rho_{01}$. This represents the opposite limit. In the $V$ system, $\rho_{02}$ decays with $\rho_{01}$ and this quenches the emission. In both cases, the control pulse also abruptly modifies the population of $|0\rangle$ during the emission, which generates a sharp feature in the emission and adds oscillations to the entire probe pulse spectrum that are discussed in Appendix B.

These results are consistent with pump-probe-control experimental results. After the probe pulse measures gain, the spectrometer integrates over the emission without temporal information. These results show that the control pulse can quench or redirect the remaining emission depending on the coherence time of the $X^{2} \Sigma_{\mathrm{g}}^{+}$and $A^{2} \Pi_{\mathrm{u}}$ states, which reduces the intensity at the on-axis detector. The control pulse influences the $\mathrm{N}_{2}^{+}$air laser pulse duration, spectrum, and direction.

\section{Summary}

In conclusion, we performed pump-probe experiments on the nitrogen molecular cation but labeled the excitation pulse "control" for clarity. Rotational excitation by the control pulse removed the initial rotational wave packet from the $B^{2} \Sigma_{\mathrm{u}}^{+}$ state. We never observed a strong rotational wave packet in the $X^{2} \Sigma_{\mathrm{g}}^{+}$state. We proposed that this may be due to coupling around $800 \mathrm{~nm}$ by the pump and control pulses that mixes the $X^{2} \Sigma_{\mathrm{g}}^{+}$and $A^{2} \Pi_{\mathrm{u}}$ states. Our measurements led to a three-level $V$-system model. It showed that coherent emission following the probe pulse is quenched or modified by the control pulse coupling the ground and middle states. Our measurement showed that emission is indeed interrupted by the control pulse.

Removing the initial rotational wave packet generates a smoother gain decay, but this has consequences for the emission. The emission also contains structure because of modulated gain during propagation and beating between transitions from different rotational states [4,26,36], but the control pulse should also smooth the temporal profile of the emission. In addition, the ability to quench or coherently redirect the emission allows the control pulse to tune the characteristics of the air laser, which could be achieved from a standoff distance. The control pulse spatial profile, temporal shape, and polarization add more degrees of freedom to craft desired behavior.

These results support the evidence that the $A^{2} \Pi_{\mathrm{u}}$ state is an important population sink that enables $\mathrm{N}_{2}^{+}$air lasing $[2,3,11]$. An obvious next step is to change the pump wavelength away from $X^{2} \Sigma_{\mathrm{g}}^{+}$to $A^{2} \Pi_{\mathrm{u}}$ state transitions so tunnel ionization establishes the initial populations in the ion. Then, only the control pulse will couple them, and it can manufacture the inversion using the $A^{2} \Pi_{\mathrm{u}}$ state dynamics.

\section{ACKNOWLEDGMENTS}

The authors are grateful for discussions with Michael Spanner, Misha Ivanov, Felipe Morales, Maria Richter, Pavel Polynkin, and Andrei Naumov. This research is supported by the U.S. Army Research Office through Grant No. W911NF14-1-0383.

\section{APPENDIX A: EXPERIMENT DETAILS}

The experimental setup adds the control pulse to a previous setup $[18,19]$. The input laser $(800 \mathrm{~nm})$ is split into three paths to form two Mach-Zehnder-type interferometers. Linear translation stages change the length of two paths to set the delay of the probe and control pulses relative to the pump pulse. Zero probe and control delays correspond to the arrival of the pump pulse in the focus. A $200-\mu \mathrm{m}$-thick beta barium borate crystal in the probe path frequency-doubles the probe pulse, and spectral filters isolate the second harmonic from the fundamental. A dichroic mirror recombines the probe pulse with the pump and control pulses. A half wave plate and polarizer in each path control the intensity and polarization of each pulse.

The polarizations of the pump and probe are linear and parallel. A quarter wave plate in the control path changes the polarization of the control pulse to near circular $(\epsilon \approx 0.7)$ after the polarizer. The three pulses are collinear. In this geometry, molecular alignment is different during the revival of pump- and control-induced rotational wave packets. The circularly polarized control pulse generates alignment along the propagation direction during the revival [37], while the pump pulse generates alignment along the polarization direction during the revival. In both cases, the rotational wave packets modulate alignment in three dimensions. The probe pulse is sensitive to alignment along the polarization direction.

A concave mirror $(f / 30)$ focuses the pulses into the $\mathrm{N}_{2}$ gas jet in vacuum. A $200-\mu \mathrm{m}$-wide sonic nozzle and a pulsed valve with a backing pressure of $\approx 6$ bars of nitrogen gas generate the supersonic jet. Operating pressure in the chamber is $\approx 10^{-5}$ mbar. Three linear stages control the position of the nozzle relative to the laser in the vacuum chamber. The pump pulse ionizes $\mathrm{N}_{2}$ and creates a plasma channel in the jet. A nearby conductive mesh measures ionization, which is maximum at the location of the focus. The nozzle is $\approx 250 \mu \mathrm{m}$ upstream from the focus, so the nozzle enclosure does not obscure the laser. The pump pulse creates high harmonics in the focus, which are collected on an XUV spectrometer in vacuum. The cutoff in the high harmonic spectrum provides the pump pulse intensity. The pump intensity determines the intensities of probe and control pulses using their relative energy, duration, and size.

The probe pulse is amplified after the pump pulse ionizes the jet. A mirror on a translation stage directs the laser out of the vacuum chamber. Absorption filters, interference filters, and dichroic mirrors isolate the probe pulse. A lens refocuses the probe onto a conventional UV/Vis fiber spectrometer (Ocean Optics Maya Pro 2000) that measures the spectrum. The amplification ratio is the intensity integrated over the peak in the spectrum that roughly corresponds to the $\mathrm{P}$ branch at $391 \mathrm{~nm}\left(I_{\text {out }}\right)$, which is divided by a reference value of the 

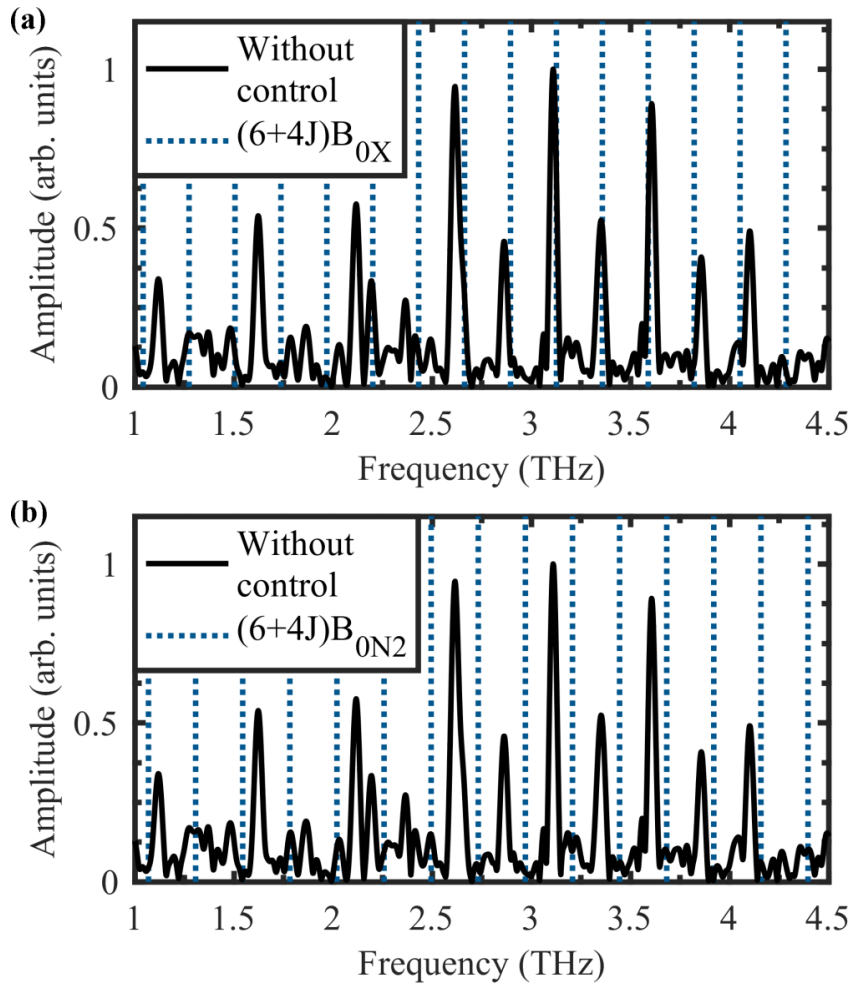

FIG. 5. Amplitude of the Fourier transform of the modulations as a function of frequency without a control pulse. The vertical dashed lines indicate the rotational frequencies for the ground state of (a) the ion and (b) the neutral molecule. $I_{\text {pump }}=2.5 \times 10^{14} \mathrm{~W} / \mathrm{cm}^{2}$.

integrated intensity with no gain present $\left(I_{\text {in }}\right)$. The natural logarithm of the amplification ratio is the gain-length product:

$$
g_{L}=\ln \left(\frac{I_{\text {out }}}{I_{\text {in }}}\right) .
$$

The modulations are isolated from decaying $g_{L}$ by fitting and subtracting an exponential decay function. The modulations are multiplied by a broad sinusoidal function, so their amplitude smoothly decreases to zero at the start and end, and both ends are padded with zeros to improve the Fourier transforms. The Fourier transforms of pump-control-probe measurements only include data from probe delays after the control delay. The rotational wave packet in the $B^{2} \Sigma_{\mathrm{u}}^{+}$state is the dominant contribution to the modulations superimposed on the gain decay as shown in Fig. 2(a), but Fig. 5 makes the same comparison for the two states with relatively poor agreement: the ground state of the ion in (a) and the neutral molecule in (b).

When the control pulse exchanges population between the $X^{2} \Sigma_{\mathrm{g}}^{+}$and $A^{2} \Pi_{\mathrm{u}}$ states, there is a control-induced change of gain due to the population transfer involving the ground state. Figure 6(a) shows gain at a fixed control delay and three later probe delays (i.e., pump-control-probe). The control-induced change is apparent as the intensity increases. There is an upward trend as a function of intensity, in addition to a dip at higher intensity. The dip is likely from modified rotational wave packets, as it appears at intensities where the control pulse begins to modify modulations. Overall, gain is slightly

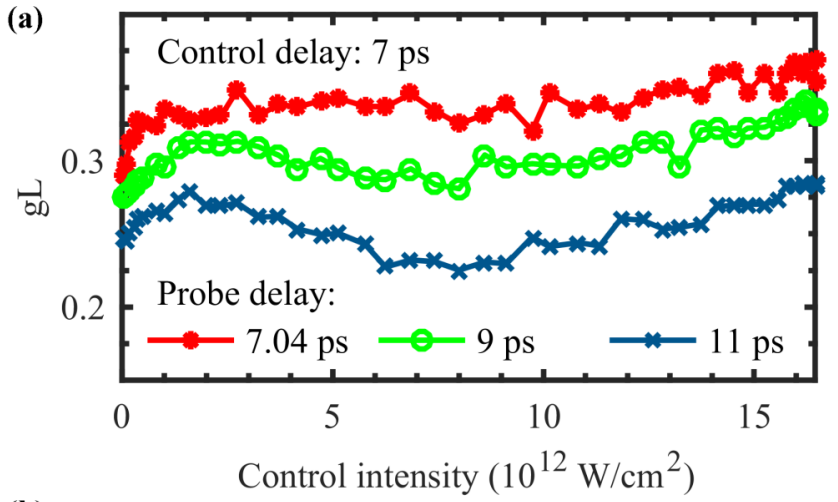

(b)

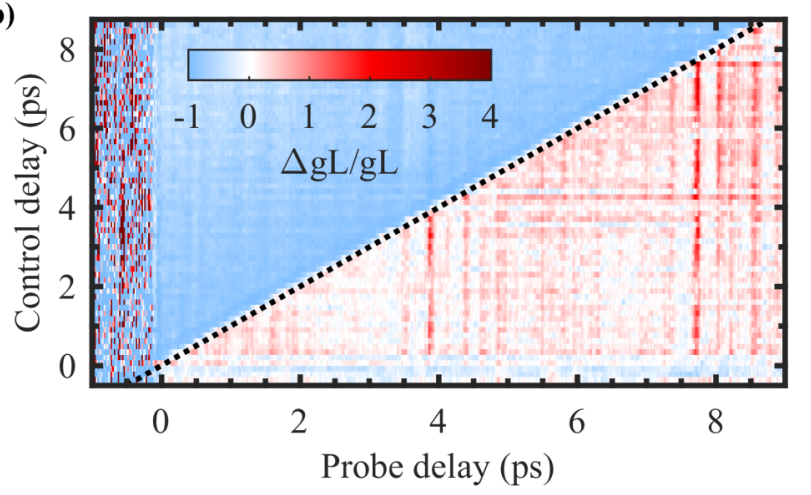

FIG. 6. (a) Gain at three probe delays as a function of control intensity after the control pulse interaction. Gain at the lowest control intensity is approximately equal to gain with no control pulse. $I_{\text {pump }}=1.9 \times 10^{14} \mathrm{~W} / \mathrm{cm}^{2}$. (b) The relative difference between gain with the control pulse and without control as a function of control and probe delay. $I_{\text {pump }}=2.5 \times 10^{14} \mathrm{~W} / \mathrm{cm}^{2}, I_{\text {control }}=10^{13} \mathrm{~W} / \mathrm{cm}^{2}$.

higher after interacting with the control pulse, but this also depends on control delay.

Figure 6(b) shows the control-induced change to gain

$$
\Delta g_{L} / g_{L}=\frac{g_{L \text { control }}-g_{L}}{g_{L}}
$$

as a function of probe and control delay, which is the relative difference between gain with the control pulse $\left(g_{L \text { control }}\right)$ and without it $\left(g_{L}\right)$. The diagonal line is the separation between pump-probe-control (top) and pump-control-probe (bottom) measurements. The negative top region is modified emission. The pump-control-probe region is mostly positive with additional structure, so gain is higher on average in the presence of the control pulse at this intensity.

\section{APPENDIX B: CALCULATION DETAILS}

The calculation considers three states in a $\mathrm{V}$ configuration $(|0\rangle,|1\rangle$, and $|2\rangle)$. The Hamiltonian for this system is $H=$ $H_{0}+V(t)$, where

$$
H_{0}=\left(\begin{array}{ccc}
0 & 0 & 0 \\
0 & \epsilon_{1} & 0 \\
0 & 0 & \epsilon_{2}
\end{array}\right)
$$


and

$$
V(t)=\left(\begin{array}{ccc}
0 & -\mu_{01} E_{C}(t) & -\mu_{02} E_{P}(t) \\
-\mu_{01} E_{C}(t) & 0 & 0 \\
-\mu_{02} E_{P}(t) & 0 & 0
\end{array}\right) .
$$

In the $\mathrm{N}_{2}{ }^{+}$system, the transition dipole moments for the $B^{2} \Sigma_{\mathrm{u}}^{+}$to $X^{2} \Sigma_{\mathrm{g}}^{+}$transition and the $A^{2} \Pi_{\mathrm{u}}$ to $X^{2} \Sigma_{\mathrm{g}}^{+}$transition are parallel and perpendicular, respectively. To compare with the calculated system, we effectively assume that the probe and control pulses have perpendicular polarization and that the molecules are constantly aligned with the probe polarization at a fixed internuclear distance.

The calculation uses atomic units (a.u.). The dipole moments are $\mu_{01}=0.26$ a.u. and $\mu_{02}=0.74$ a.u., and the state energies are $\epsilon_{1}=1.54 \mathrm{eV}$ and $\epsilon_{2}=3.17 \mathrm{eV}$ [30]. The initial electric field of the probe $\left(E_{P}\right)$ and control $\left(E_{C}\right)$ pulses is a transform-limited Gaussian pulse multiplied by a cosine function and set to zero past the first cosine zero crossing. The cosine function provides a smooth decrease to zero, so the free-induction decay is not seeded. The peak intensity of the probe pulse is $I_{P, 0}=10^{8} \mathrm{~W} / \mathrm{cm}^{2}$ and the control pulse is $I_{C, 0}=4 \times 10^{12} \mathrm{~W} / \mathrm{cm}^{2}$. Their full width at half maximum duration is $100 \mathrm{fs}$ and the field is zero beyond $\pm 400 \mathrm{fs}$. The center wavelengths of the probe $(393 \mathrm{~nm})$ and control $(800 \mathrm{~nm})$ pulses are detuned by a couple of nanometers from each transition. The time grid spans from -0.5 to $15 \mathrm{ps}$ using a time step of 20 as.

The Liouville-von Neumann equation $i \hbar \dot{\rho}=[H, \rho]$ determines the evolution of the density matrix $(\rho)$ in time. The initial populations are $\rho_{00,0}=0.2, \rho_{11,0}=0.1$, and $\rho_{22,0}=$ 0.7 , and population decays to the ground state on the timescale of $T_{1}=10 \mathrm{~ns}$. The results are qualitatively similar for a freeinduction decay due to absorption when the system is not inverted. The off-diagonal density-matrix elements are the coherence between states, so they are initially zero. While a preceding pump pulse may establish coherence between the $|0\rangle$ and $|1\rangle$ states, we separately included this coherence as an initial condition and obtained the same conclusions. We change the coherence decay time for the $|0\rangle$ and $|1\rangle$ states $\left(T_{2}^{01}\right)$ to observe different behaviors. The Crank-Nicolson method in time provides a numerical solution to the equations of motion for the density-matrix elements [38].

The material polarization $P_{\mu}=N \mu_{02}\left(\rho_{02}+\rho_{20}\right)$, where $N$ is the number density, is a source term for the probe pulse propagation using the one-direction decomposition of the wave equation in the reference frame of the probe pulse:

$$
\frac{\partial E_{P}(t, z)}{\partial z}=-\left(\frac{1}{c}-\frac{1}{v_{g}}\right) \frac{\partial E_{P}(t, z)}{\partial t}-\frac{2 \pi}{c} \frac{\partial P_{\mu}(t, z)}{\partial t},
$$

where $v_{g}$ is the group velocity. Similarly, $\rho_{01}$ is included in the control pulse propagation. The propagation step of $1 \mu \mathrm{m}$ in a density of $10^{17} \mathrm{~cm}^{-3}$ creates a small free-induction decay.

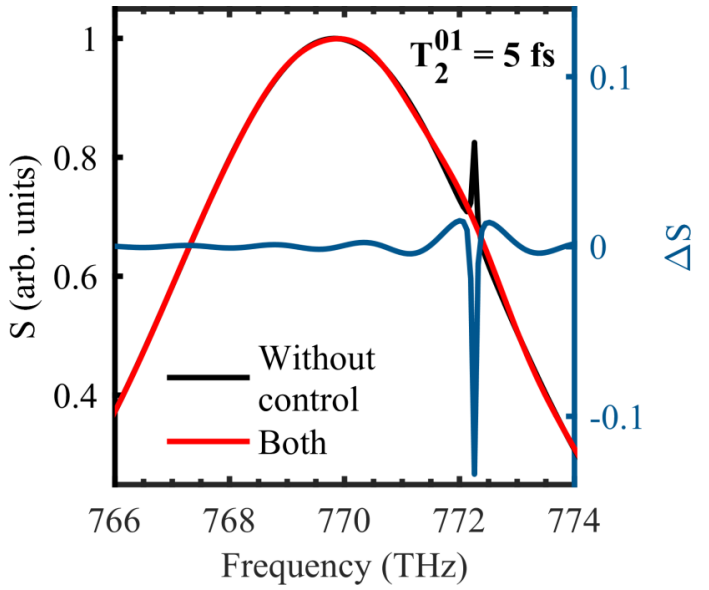

FIG. 7. The calculated spectrum of the probe pulse with and without the control pulse, and the difference on a separate axis $(\Delta S)$.

Propagation includes first- and second-order changes to the electric field and is suitable for many propagation steps, but the calculation uses a single step to accommodate the computation time required for many near-field positions.

The probe and control pulses are collinear and have beam waists of 50 and $75 \mu \mathrm{m}$, respectively. The near-field radial axis has 500 positions and extends to $499 \mu \mathrm{m}$. The particular radial positions enable an efficient implementation of the Hankel transform, which converts the electric field from the near-field radial position to the far-field divergence at each time step [39]. The emission is calculated for the first 200 positions, while the remaining are padded with zeros. The closest on-axis radial position is $0.8 \mu \mathrm{m}$. The far-field divergence axis assumes the transition frequency corresponding to $391 \mathrm{~nm}$ but depends on frequency in general. This is a reasonable assumption for the free-induction decay. The Hilbert transform provides the electric-field envelope, which improves the appearance of figures.

The calculation showed that population transfer between $|0\rangle$ and $|1\rangle$ modifies the coherence between $|0\rangle$ and $|2\rangle$ independent of $T_{2}^{01}$, but $T_{2}^{01}$ determines whether the emission is coherently redirected or quenched. When coherent population transfer dominates, the emission contains a phase shift in time that corresponds to a different line shape in frequency [40]. Other effects can also redirect and quench the emission, like the Stark shift of the levels by the control pulse [35]. If the control pulse was detuned from resonances, Rabi flopping and Stark shifts by the control pulse would both be important contributions. Regardless of the coherence time, gain is abruptly modified during the emission. This generates a sharp feature in the emission that corresponds to broader spectral bandwidth. As a result, the amplified spectrum contains oscillations that are highlighted in Fig. 7. The oscillations can be described as spectral interference between the original emission and the control-induced change to the emission.
[1] J. Yao, B. Zeng, H. Xu, G. Li, W. Chu, J. Ni, H. Zhang, S. L. Chin, Y. Cheng, and Z. Xu, High-brightness switchable multiwavelength remote laser in air, Phys. Rev. A 84, 051802(R) (2011).
[2] H. Xu, E. Lötstedt, A. Iwasaki, and K. Yamanouchi, Sub-10-fs population inversion in $\mathrm{N}_{2}{ }^{+}$in air lasing through multiple state coupling, Nat. Commun. 6, 8347 (2015). 
[3] J. Yao, S. Jiang, W. Chu, B. Zeng, C. Wu, R. Lu, Z. Li, H. Xie, G. Li, C. Yu, Z. Wang, H. Jiang, Q. Gong, and Y. Cheng, Population Redistribution Among Multiple Electronic States of Molecular Nitrogen Ions in Strong Laser Fields, Phys. Rev. Lett. 116, 143007 (2016).

[4] X. Zhong, Z. Miao, L. Zhang, Q. Liang, M. Lei, H. Jiang, Y. Liu, Q. Gong, and C. Wu, Vibrational and electronic excitation of ionized nitrogen molecules in intense laser fields, Phys. Rev. A 96, 043422 (2017).

[5] M. Richter, F. Morales, M. Spanner, O. Smirnova, and M. Ivanov, Optical lasing during laser filamentation in the nitrogen molecular ion: Ro-vibrational inversion, in Proceedings of the 2017 Conference on Lasers and Electro-Optics Europe European Quantum Electronics Conference (CLEO/Europe-EQEC) (Munich, 2017), p. CG_P_15.

[6] B. Xu, S. Jiang, J. Yao, J. Chen, Z. Liu, W. Chu, Y. Wan, F. Zhang, L. Qiao, R. Lu, Y. Cheng, and Z. Xu, Free-space $\mathrm{N}_{2}{ }^{+}$ lasers generated in strong laser fields: the role of molecular vibration, Opt. Express 26, 13331 (2018).

[7] H. Li, M. Hou, H. Zang, Y. Fu, E. Lötstedt, T. Ando, A. Iwasaki, K. Yamanouchi, and H. Xu, Significant Enhancement of $\mathrm{N}_{2}{ }^{+}$Lasing by Polarization-Modulated Ultrashort Laser Pulses, Phys. Rev. Lett. 122, 013202 (2019).

[8] Y. Zhang, E. Lötstedt, and K. Yamanouchi, Mechanism of population inversion in laser-driven $\mathrm{N}_{2}{ }^{+}$, J. Phys. B 52, 055401 (2019).

[9] A. Zhang, M. Lei, J. Gao, C. Wu, Q. Gong, and H. Jiang, Subfemtosecond-resolved modulation of superfluorescence from ionized nitrogen molecules by 800 -nm femtosecond laser pulses, Opt. Express 27, 14922 (2019).

[10] T. Ando, E. Lötstedt, A. Iwasaki, H. Li, Y. Fu, S. Wang, H. $\mathrm{Xu}$, and K. Yamanouchi, Rotational, Vibrational, and Electronic Modulations in $\mathrm{N}_{2}{ }^{+}$lasing at $391 \mathrm{~nm}$ : Evidence of Coherent $B^{2} \Sigma_{u}^{+}-X^{2} \Sigma_{g}^{+}-A^{2} \Pi_{u}$ Coupling, Phys. Rev. Lett. 123, 203201 (2019).

[11] Y. Wan, B. Xu, J. Yao, J. Chen, Z. Liu, F. Zhang, W. Chu, and Y. Cheng, Polarization ellipticity dependence of $\mathrm{N}_{2}{ }^{+}$air lasing: The role of coupling between the ground state and a photo-excited intermediate state, J. Opt. Soc. Am. B 36, G57 (2019).

[12] Q. Zhang, H. Xie, G. Li, X. Wang, H. Lei, J. Zhao, Z. Chen, J. Yao, Y. Cheng, and Z. Zhao, Sub-cycle coherent control of ionic dynamics via transient ionization injection, Commun. Phys. 3, 50 (2020).

[13] H. Xie, Q. Zhang, G. Li, X. Wang, L. Wang, Z. Chen, H. Lei, and Z. Zhao, Vibrational population transfer between electronic states of $\mathrm{N}_{2}^{+}$in polarization-modulated intense laser fields, Phys. Rev. A 100, 053419 (2019).

[14] Y. Fu, E. Lötstedt, H. Li, S. Wang, D. Yao, T. Ando, A. Iwasaki, F. H. M. Faisal, K. Yamanouchi, and H. Xu, Optimization of $\mathrm{N}_{2}{ }^{+}$lasing through population depletion in the $X^{2} \Sigma_{g}{ }^{+}$ state using elliptically modulated ultrashort intense laser fields, Phys. Rev. Research 2, 012007 (2020).

[15] A. Zhang, Q. Liang, M. Lei, L. Yuan, Y. Liu, Z. Fan, X. Zhang, S. Zhuang, C. Wu, Q. Gong, and H. Jiang, Coherent modulation of superradiance from nitrogen ions pumped with femtosecond pulses, Opt. Express 27, 12638 (2019).

[16] A. Mysyrowicz, R. Danylo, A. Houard, V. Tikhonchuk, X. Zhang, Z. Fan, Q. Liang, S. Zhuang, L. Yuan, and Y. Liu, Lasing without population inversion in $\mathrm{N}_{2}{ }^{+}$, APL Photon. 4, 110807 (2019)

[17] J. Chen, J. Yao, H. Zhang, Z. Liu, B. Xu, W. Chu, L. Qiao, Z. Wang, J. Fatome, O. Faucher, C. Wu, and Y. Cheng, Electroniccoherence-mediated molecular nitrogen-ion lasing in a strong laser field, Phys. Rev. A 100, 031402(R) (2019).

[18] M. Britton, P. Laferrière, D. H. Ko, Z. Li, F. Kong, G. Brown, A. Naumov, C. Zhang, L. Arissian, and P. B. Corkum, Testing the Role of Recollision in $\mathrm{N}_{2}{ }^{+}$Air Lasing, Phys. Rev. Lett. 120, 133208 (2018).

[19] M. Britton, M. Lytova, P. Laferrière, P. Peng, F. Morales, D. H. Ko, M. Richter, P. Polynkin, D. M. Villeneuve, C. Zhang, M. Ivanov, M. Spanner, L. Arissian, and P. B. Corkum, Short- and long-term gain dynamics in $\mathrm{N}_{2}{ }^{+}$air lasing, Phys. Rev. A 100, 013406 (2019).

[20] D. Kartashov, S. Haessler, S. Ališauskas, G. Andriukaitis, A. Pugžlys, A. Baltuška, J. Möhring, D. Starukhin, M. Motzkus, A. Zheltikov, M. Richter, F. Morales, O. Smirnova, M. Y. Ivanov, and M. Spanner, Transient inversion in rotationally aligned nitrogen ions in a femtosecond filament, in Research in Optical Sciences (Optical Society of America, Washington, 2014), p. HTh4B.5.

[21] M. Richter, M. Lytova, F. Morales, S. Haessler, O. Smirnova, M. Spanner, and M. Ivanov, Rotational quantum beat lasing without inversion, Optica 7, 586 (2020).

[22] H. Zhang, C. Jing, J. Yao, G. Li, B. Zeng, W. Chu, J. Ni, H. Xie, H. Xu, S. L. Chin, K. Yamanouchi, Y. Cheng, and Z. Xu, Rotational Coherence Encoded in an "Air-Laser" Spectrum of Nitrogen Molecular Ions in an Intense Laser Field, Phys. Rev. X 3, 041009 (2013).

[23] B. Zeng, W. Chu, G. Li, J. Yao, H. Zhang, J. Ni, C. Jing, H. $\mathrm{Xie}$, and Y. Cheng, Real-time observation of dynamics in rotational molecular wave packets by use of air-laser spectroscopy, Phys. Rev. A 89, 042508 (2014).

[24] H. Xie, B. Zeng, G. Li, W. Chu, H. Zhang, C. Jing, J. Yao, J. $\mathrm{Ni}$, Z. Wang, Z. Li, and Y. Cheng, Coupling of $\mathrm{N}_{2}{ }^{+}$rotational states in an air laser from tunnel-ionized nitrogen molecules, Phys. Rev. A 90, 042504 (2014).

[25] M. Lei, C. Wu, A. Zhang, Q. Gong, and H. Jiang, Population inversion in the rotational levels of the superradiant $\mathrm{N}_{2}{ }^{+}$pumped by femtosecond laser pulses, Opt. Express 25, 4535 (2017).

[26] X. Zhong, Z. Miao, L. Zhang, H. Jiang, Y. Liu, Q. Gong, and C. Wu, Optimizing the 391-nm lasing intensity from ionized nitrogen molecules in 800-nm femtosecond laser fields, Phys. Rev. A 97, 033409 (2018).

[27] L. Arissian, B. Kamer, A. Rastegari, D. M. Villeneuve, and J.-C. Diels, Transient gain from $\mathrm{N}_{2}{ }^{+}$in light filaments, Phys. Rev. A 98, 053438 (2018).

[28] H. Xu, E. Lötstedt, T. Ando, A. Iwasaki, and K. Yamanouchi, Alignment-dependent population inversion in $\mathrm{N}_{2}{ }^{+}$in intense few-cycle laser fields, Phys. Rev. A 96, 041401(R) (2017).

[29] K. F. Lee, E. A. Shapiro, D. M. Villeneuve, and P. B. Corkum, Coherent creation and annihilation of rotational wave packets in incoherent ensembles, Phys. Rev. A 73, 033403 (2006).

[30] F. R. Gilmore, R. R. Laher, and P. J. Espy, Franck-Condon factors, r-centroids, electronic transition moments, and einstein coefficients for many nitrogen and oxygen band systems, J. Phys. Chem. Ref. Data 21, 1005 (1992). 
[31] J. Yao, G. Li, C. Jing, B. Zeng, W. Chu, J. Ni, H. Zhang, H. Xie, C. Zhang, H. Li, H. Xu, S. L. Chin, Y. Cheng, and Z. Xu, Remote creation of coherent emissions in air with two-color ultrafast laser pulses, New J. Phys. 15, 023046 (2013).

[32] G. Li, C. Jing, B. Zeng, H. Xie, J. Yao, W. Chu, J. Ni, H. Zhang, H. Xu, Y. Cheng, and Z. Xu, Signature of superradiance from a nitrogen-gas plasma channel produced by strong-field ionization, Phys. Rev. A 89, 033833 (2014).

[33] Y. Liu, P. Ding, G. Lambert, A. Houard, V. Tikhonchuk, and A. Mysyrowicz, Recollision-Induced Superradiance of Ionized Nitrogen Molecules, Phys. Rev. Lett. 115, 133203 (2015).

[34] Z. Li, F. Kong, G. Brown, T. Hammond, D.-H. Ko, C. Zhang, and P. B. Corkum, Perturbing laser field dependent high harmonic phase modulations, J. Phys. B 51, 125601 (2018).

[35] S. Bengtsson, E. W. Larsen, D. Kroon, S. Camp, M. Miranda, C. L. Arnold, A. L'Huillier, K. J. Schafer, M. B. Gaarde, L. Rippe, and J. Mauritsson, Space-time control of free induction decay in the extreme ultraviolet, Nat. Photon. 11, 252 (2017).
[36] Z. Miao, X. Zhong, L. Zhang, W. Zheng, Y. Gao, Y. Liu, H. Jiang, Q. Gong, and C. Wu, Stimulated-raman-scatteringassisted superfluorescence enhancement from ionized nitrogen molecules in 800-nm femtosecond laser fields, Phys. Rev. A 98, 033402 (2018).

[37] C. T. L. Smeenk and P. B. Corkum, Molecular alignment using circularly polarized laser pulses, J. Phys. B 46, 201001 (2013).

[38] J. Crank and P. Nicolson, A practical method for numerical evaluation of solutions of partial differential equations of the heat-conduction type, Math. Proc. Camb. Philos. Soc. 43, 50 (1947).

[39] M. Guizar-Sicairos and J. C. Gutiérrez-Vega, Computation of quasi-discrete Hankel transforms of integer order for propagating optical wave fields, J. Opt. Soc. Am. A 21, 53 (2004).

[40] C. Ott, A. Kaldun, P. Raith, K. Meyer, M. Laux, J. Evers, C. H. Keitel, C. H. Greene, and T. Pfeifer, Lorentz meets Fano in spectral line shapes: A universal phase and its laser control, Science 340, 716 (2013). 